\title{
Hidradenitis suppurativa treated with combination of infliximab and dapsone
}

\author{
Kozub P, Simaljakova M \\ Department of Dermatovenerology, University Hospital Bratislava and Faculty of Medicine, Comenius University, \\ Bratislava, Slovakia. peter.kozub@laposte.net
}

\begin{abstract}
Hidradenitis suppurativa (HS) is chronic inflammatory disease characterized by recurrent painful nodules, which leads to forming an abscess and sinus tracts, together with pustulation and hypertrophic scarring in the areas of apocrine sweat glands. Systemic and local antibiotic therapy, together with corticoid therapy, sulphonates, retinoids and surgical treatment with incisions and drainage belongs to actual treatment possibilities. Biological therapy represents the absolute peak in the treatment last years, mostly TNFa-inhibitors. Even though their very good short term effect is described, long term keeping up the good long effect of the treatment is not achieved with satisfaction. This is the reason to reach for traditional therapeutic possibilities combined with biological treatment in cases of torpid and resistive disease. In the following article a case report of a patient with hidradenitis suppurativa treated subsequently with combination of TNFa-inhibitors and dapson (Fig. 6, Ref. 19). Full Text in PDF www.elis.sk.

Key words: hidradenitis suppurativa, therapy, TNFa inhibitors, infliximab, sulphonates, dapson.
\end{abstract}

Hidradenitis suppurativa (HS) is chronic inflammatory disease characterized by recurrent painful nodules, which leads to forming an abscess and sinus tracts, together with pustulation and hypertrophic scarring in the areas of apocrine sweat glands. It is spread evenly between both genders. Obesity and smoking belong to risk factors. Hormonal factors are also described, which is supported by the fact, that the disease can be mitigated in gravidity. Various infections can worsen the existing manifestations. In clinical findings painful nodules and fistulas draining pus are seen. Predilection areas of the disease are the areas with apocrine glands (axillar and inguinal areas, perigenital and perianal area, submammar space), ectopic nodules can be located anywhere on the body. Sanation is accompanied with forming of hypertrophic scars, which can lead to dermogenous contractures. Systemic and local antibiotic therapy, together with corticoid therapy, sulfones, retinoids and surgical treatment with incisions and drainage belongs to actual treatment possibilities. Complete restoration is in the present possible only by removing the skin with apocrinne sweat glands in the full thickness, which can result in cosmetic disturbing features, dermogennous contractures and with predisposed individuals the new focus can appear in other part rich in apocrine sweat glands. Biologics, mainly TNF $\alpha$-inhibitors, are even more often finding their place in the treatment of other diseases, where an important role is played by tumor necrosis factor alpha $(\mathrm{TNF} \alpha)$. Hidradenitis suppurativa is one of these diseases.

Department of Dermatovenerology, University Hospital Bratislava and Faculty of Medicine, Comenius University, Bratislava, Slovakia

Address for correspondence: P. Kozub, MD, Department of Dermatovenerology, University Hospital Bratislava and Faculty of Medicine, Comenius University, Mickiewiczova 13, SK-813 69 Bratislava, Slovakia.

\section{Case report}

53-years old female with two years history of hidradenitis suppurativa was admited at the Clinic of Dermatovenerology in Bratislava in March 2009. Painful nodules and many fistulas in the medial part of thighs (Figs 1 and 2), left gluteal and submammar area were dominating the clinical findings, ectopic focuses were found on the front side of the body. Axillar, perigenital and inguinal areas were not affected. Patient repeatedly underwent incisions and drainages at surgical ambulance, was treated with various systemic antibiotics (doxycyclin, cefotaxim, ciprofloxacin) and isoprinosin, but with no significant effect. Family history was insignificant. The patient was also suffering from untreated diabetes mellitus, diabetologist recommended dietetic approach. Patient smoked up to 15 cigarettes a day for many years. During the hospitalization, isotretionin was dosed $0.8 \mathrm{mg} / \mathrm{kg} / \mathrm{day}$. Patient used the medication also after leaving the hospital, but suppression of disease manifestations occurred only partialy and thus the treatment was terminated after 6 months.

In September 2009 biological treatment with infliximab was induced. Patient with $92 \mathrm{~kg}$ was dosed with $500 \mathrm{mg}$ of infliximab in 0th, 2nd, and sixth week and then every 8 weeks. After a week the pustulant secernation was reduced by half and the pain was significantly reduced. After the second infusion some nodules regressed, but also a few new appeared. At the end of induction phase (6th week) the effect was satisfying. Clinical evaluation of the treatment (HSS - Hidradenitis Suppurativa Score, modified version according to Sartorius) was used in the 22 nd week. We were directed more with subjective feelings of the patient (pain), clinical findings (number of nodules, pus secernation) and inflammatory blood markers (erythrocyte sedimentation rate, 


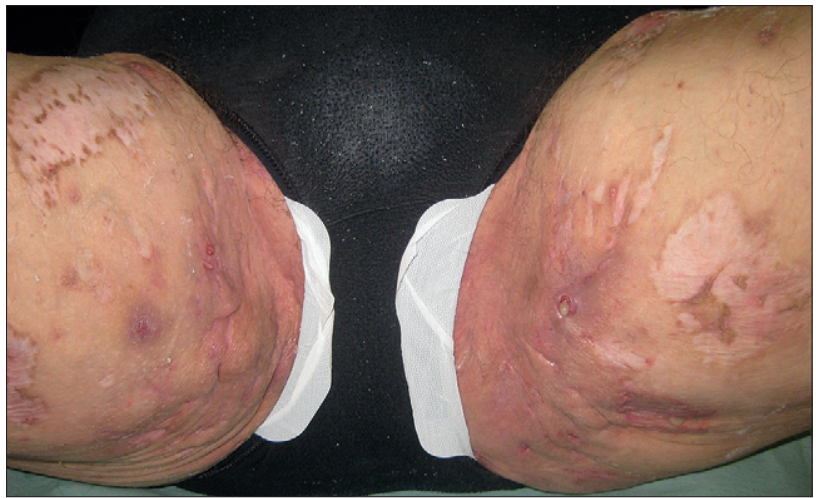

Fig. 1. Before treatment.

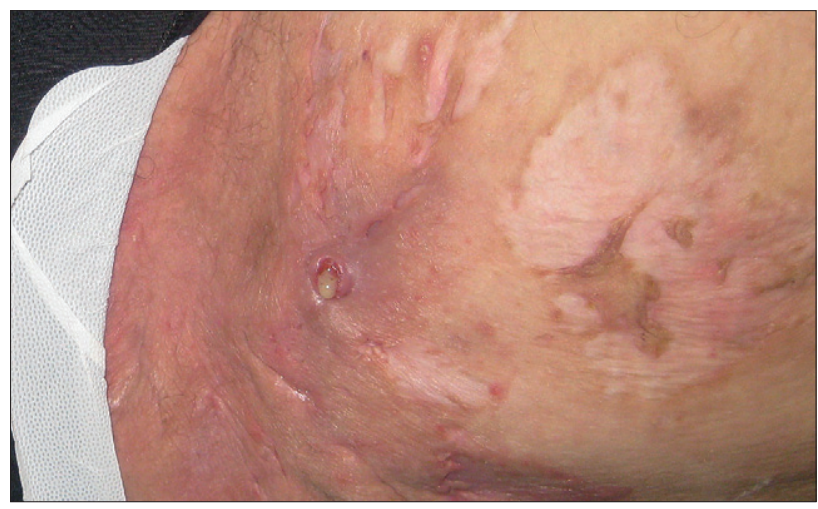

Fig. 2. Detail of right thighs (before treatment).

CRP

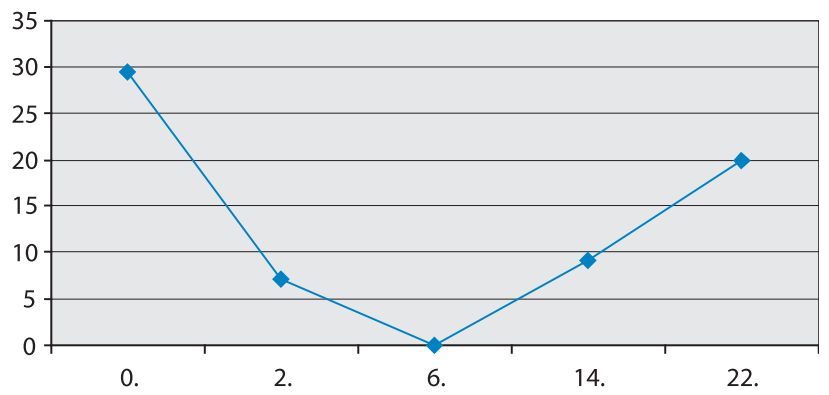

Fig. 3. Changes in CRPlevels in first 22 weeks of treatment with infliximab.

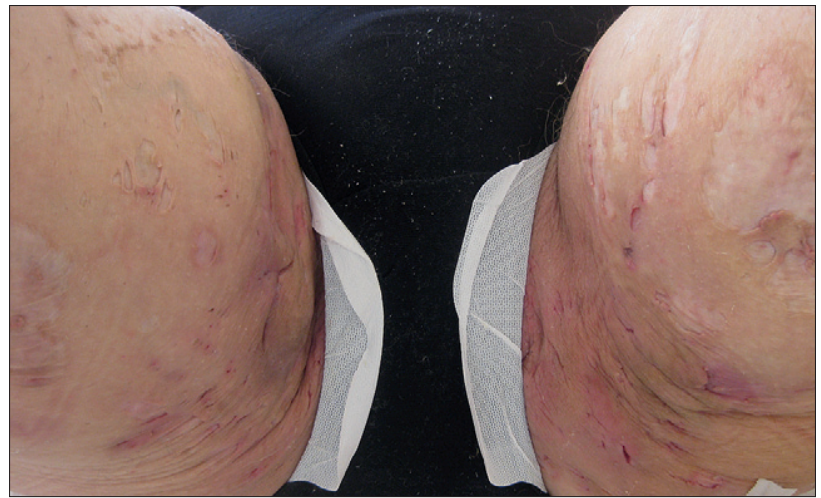

Fig. 5. 43th week of treatment.

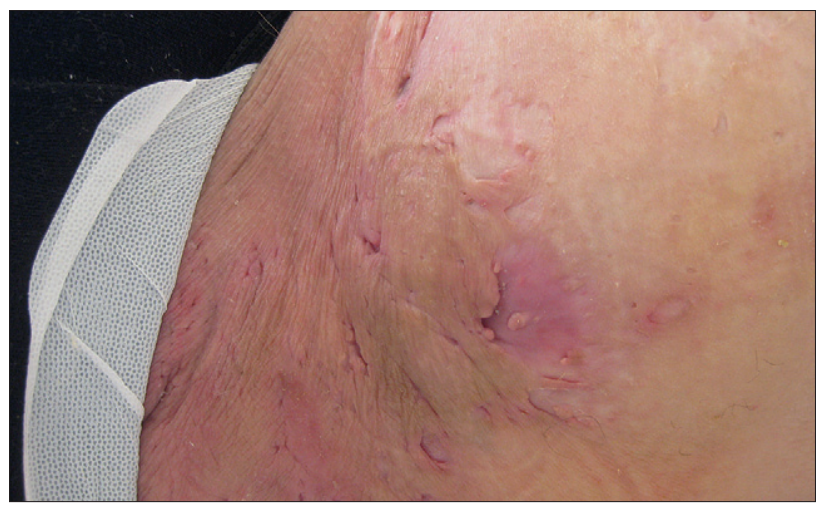

Fig. 6. Detail of right thighs (43th week of treatment).

leukocytosis, CRP). As main objective parameter of the disease C-reactive protein (CRP) was selected. After the initial decrease and following normalization of values at the end of the induction phase normal levels of CRP were not possible to be sustained during the first two maintenance intervals. CRP levels were rising at the end of the first and second week of the 8 week period, which correlated with clinical findings. The changes in CRP during the first 22 weeks of treatment are shown in Figure 3.

Patient referred to the treatment as the best she received so far, but she was not satisfied. No side effects were mentioned. No

CRP

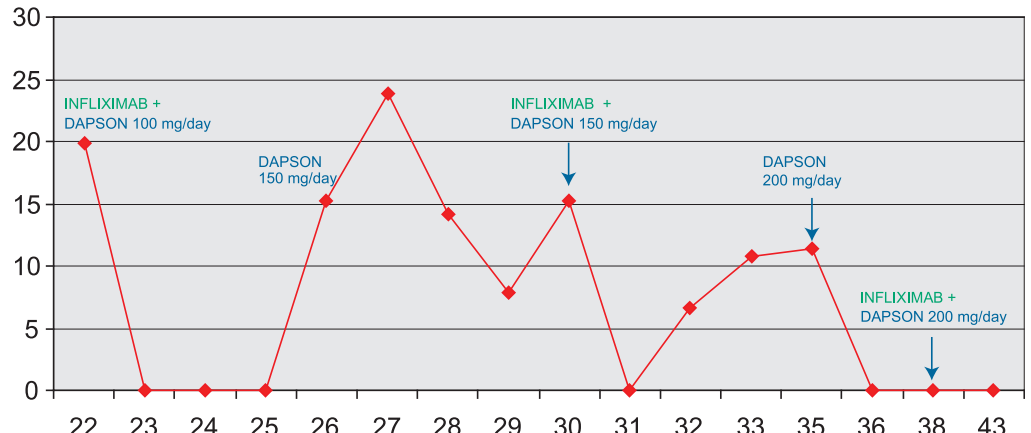

Fig. 4. Changes in CRP levels between 22th - 43th week of combined therapy with infliximab and dapson (the value of CRP $<5$ is considered normal, for better view, CRP level of zero is shown as normal in this graph). 
other medication was taken, she was not ill either. That was why we decided to add dapsone in initial dose $1 \mathrm{mg} / \mathrm{kg} /$ day (100 mg/day) in the 22nd week. The manifestations started to alliviate quickly, pain and secernation subsided. Changes of CRP were observed in weekly intervals (Fig. 4) and based on them the dose of dapsone was adjusted. CRP was normal during the first three weeks of combined therapyand that was mirrored in clinical findings manifestations on the body and medial area of the left thigh were resorbed completely. In 4th week of combined therapy deterioration of manifestations started in the medial part of right thigh and left gluteal area, elevation of CRP was recorded. Dapsone dose was adjusted to $150 \mathrm{mg} /$ day. Despite that worsening of manifestations and CRP rise followed next week. Next two weeks followed with CRP decrease, which was again connected with clinical improvement. In 30th week next infusion of infliximab was dosed. Normal levels of CRP were attained only for a week, so dapsone dose was raised to $200 \mathrm{mg}$ /day in the 13th week of combined therapy. Since then, significant improvement of the clinical findings followed, nodules were resorbed gradually, pus secernation became sporadic. Normal levels of CRP lasted until the next infusion in 38th week and were held in normal levels even in 43th week. Patient subjectively manifested only with intermittent and minimal pain. Remaining nodules were present in left gluteal area, one larger nodule in medial area of right thigh (Figs 5 and 6). Patient was has tolerated the treatment, currently is not sick, does not feel tired, levels of hemoglobin and methemoglobin are normal.

\section{Discussion}

Hidradenitis suppurativa belongs to diseases with worldwide prevalence of $1 \%$ (1). Sealing of the hair follicle in the area with apocrine sweat glands (follicular hyperkeratosis) has important role in pathogenesis of the disease, rupture of the follicle and perifolicular inflammation follows. Bacterial super infection is common. In present, there is no treatment, which could manage the disease completely in every patient. Together with incisions and draining of abscesses antibiotics are commonly used. In easier cases local antibiotics (klindamycin) with antiseptics are used, in severe cases systemic antibiotics (rifampicin, klindamicin, amoxicillin + clavulanate, tetracyclin, cephalosporin) in monotherapy or combined (rifampicine + clindamicin) are used. Metronidazol can be added in case of severe scent of the manifestations. Systemic and intralesionally applied corticoids are used. Other therapeutic possibilities are retinoids, dapson, antiandrogenes or wide radical excision followed by sanation per secundam or with transplantation. In the case of failure of the mentioned therapy biologics are applied. Hidradenitis suppurativa belongs to diseases with "offlabel" indication group for TNF $\alpha$-blockers, because it was shown that TNF $\alpha$ has an important role in pathogenesis of this disease (2).

$\mathrm{TNF} \alpha$-inhibitors (infliximab, adalimumab, etanercept) were considered as a hope for patients with hidradenitis suppurativa. Hidradenitis suppurativa can be associated with inflammatory bowel diseases (more frequently with morbus Crohn, less frequently with ulcerous colitis) (19). First favorable results were observed in patients with inflammatory bowel disease and parallel hidradenitis, which were treated with biological treatment. The most commonly used biologic cure was infliximab, thus the most cases described were in connection with this biologic treatment. More authors in case reports $(3,4,5,6)$ considering individual patients with morbus Crohn treated with infliximab describe very good effect of infliximab on the manifestations of hidradenitis suppurativa and long term remission of the disease.

Adams et al (6) describes the case of 17 year patient with ulcerous colitis and HS. The patient was dosed with infliximab in 0th, 2nd and 6th week. Five weeks after the first infusion the patient was without pain, pustulation, scent and the inflammation was reduced significantly. After five months from the first infusion the disease relapsed, so fourth infusion of infliximab was dosed with good response within a month. Next following and progress of the disease was not recorded. The plan was to treat patient with new infliximab infusion after the disease relaps. It was not recorded if monotherapy was used, or the patient used another systemic drugs, which could improve the manifestations of hidradenitis suppurativa.

Sullivan et al (7) published the series of 5 cases of patients with hidradenitis suppurativa treated with infliximab ( 1 male and 4 female) in year 2003. Each patient received one dose of infliximab $5 \mathrm{mg} / \mathrm{kg} /$ day and in the case the disease persisted, the following infusion was dosed 4-6 weeks later. One infusion was enough only for two patients for acceptable local finding. For the first one, the manifestation of the disease improved significantly, then she underwent surgical intervention (wide excision of affected area) and next infusion was not needed. The other patient showed minimal activity of the disease after first dose and thus she did not need the second dose. Other three patients received the second infusion. In addition two of them received combined therapy (infliximab + systemic drug), which they used before initiating biologic treatment. One patient was dosed for the whole time of infliximab treatment with cyclosporin (400 mg/day), Prednison ( $5 \mathrm{mg} /$ day) and other patient received Prednison $(20 \mathrm{mg} /$ day $)$ and intralesional corticoids. Despite that their response to treatment (even after two infusions) was mild. Improvement of local findings was observed after 3-7 days since the last infusion, very promptly (24 hours or less) the pain was reduced. All patients agreed on that infusion of infliximab was the most effective treatment they received so far. To this results, it is needed to say that (i) observation was limited (shortest 3 weeks, longest 6 months), (ii) only three patients received infliximab in monotherapy and (iii) nopatient developed complete remission of the disease.

Mekkes and Bos (8) evaluated series of 10 patients (4 male and 6 female, all of them were smokers) with hidradenitis suppurativa treated with infliximab in dose $5 \mathrm{mg} / \mathrm{kg}$ in 0 th, 2nd and 6th week with following observation during one year. For evaluating the disease HSS was used (hidradenitis suppurativa score) according to Sartorius. All patients showed improvement of manifestations after 2-6 weeks, but the length of this effect was different. Three patients showed long term improvement ( 2 years without recurrency) and 7 patients had recurrency within 4.3 to 13.4 months (average 8.5 months). Better response was observed when patients (two individuals) had clinically stronger inflammatory component. 
Patients with less expressive inflammation and with dominating multiple deep and epitelized sinuses and cysts (or pilonidal sinus) had shorter effect of treatment - recurrency occurred in 4.3 months in average.

Thielen et al in the year 2006 (9) described the case of man with hidradenitis suppurativa with severe elevated inflammatory markers (ESR 94, CRP 110, Leu 15.9) treated with combination of infliximab and methotrexate. Patient started with infliximab treatments in dose of $5 \mathrm{mg} / \mathrm{kg} /$ day in 0 th, 2nd and 6th week and continued with frequency of 8 weeks. In two weeks inflammatory markers decreased significantly (ESR 34, CRP 24, Leu 8.4) In the 12th week methotrexate was added in dose of $7.5 \mathrm{mg} /$ week in order to decrease the risk of production of neutralizing antibodies. Overall length of treatment was 104 weeks (2 years) and the treatment continued. In the article, authors describe only the efficient treatment and good long-lasting effect of combined therapy, but there is no mention about the patient, if he is without lesions or the treatment only improved persisting manifestations significantly. It is only assumable that the latter is tru, because the quality of patient's life (Dermatology Life Quality Index - DLQI) was improved by $80 \%$ in two years compared to the quality of life, which he had before the treatment with infliximab.

Brunasso et al (10) described the series of 7 patients (4 men and 3 female) with hidradenitis suppurativa treated with combination of infliximab and methotrexate. Infliximab was dosed 5 $\mathrm{mg} / \mathrm{kg}$ in 0 th, $2 \mathrm{nd}, 6$ th week and every 8 weeks after, methotrexate was dosed $7.5 \mathrm{mg} /$ week until the end of the treatment. Data from the two following years (114-122 weeks) were evaluated. The author focused not only on short-term, but also long-term benefit of treatment. Short term effect (induction phase, 3 months of treatment) was very good - adequate reduction of pain was $96.2 \%$, quality of life improvement (DLQI) $52 \%$ and reduction of affected area was $7 \%$. This effect could not be preserved for longer time. After 24 months the pain was reduced only by 34.8 $\%$, quality of life improved by $14.7 \%$ and affected area reduced by $1.25 \%$. New manifestations were observed in three cases after a year of treatment. One patient discontinued the treatment in 14th week because of infusion-response. 4 patients terminated the treatment because there was no further improvement. One patient was switched to adalimumab and later to entracept because of the dosage failure of infliximab. It can be conluded that continuous treatment with infliximab has very good short term effect, but long term effect in patients with hidradenitis suppurativa is reduced significantly.

Only one randomized, double blinded, placebo controlled trial was published (11), 38 patients with hidradenitis suppurativa were included. The effect of infliximab monotherapy was followed. Infliximab was administered during 22 weeks $(0.5 \mathrm{mg} / \mathrm{kg}$ in 0 th, 2nd, 6th, 14th and 22nd week) with following observation until the 52nd week. The following was observed - in what time after the end of treatment relapse will occur (relapse of disease was defined as decrease of the Hidradenitis Suppurativa Severity Index, HSSI, by more than $40 \%$ ). The trial completed only 5 patients. Three of those had a relapse of disease and two remained the good clinical response. 17 patients terminated the study because of continua- tion with active treatment. 5 patients included in "placebo" group terminated the study in first 8 weeks.

The published case report, series of cases and trial results showed that infliximab has very quick onset of effect, significantly and quickly reduces the pain of manifestations and suppuration, which improves the quality of life significantly. It requires continuous dosing. Nevertheless the 8 -week sustaining interval does not seem to be sufficient to hold the disease in remission. Not even in single case were recorded attempts to shorten the sustaining interval to 4-6 weeks. In opposite, authors tried various combinations of infliximab with traditional systemic treatment (methotrexate, cyclosporin, systemic corticoids). Most commonly it was the combination of infliximab with methotrexate. Methotrexate in monotherapy does not have significant effect in therapy of hidradenitis suppurativa (12). Its addition to therapy with infliximab was concluded by authors as a prevention of neutralizing antibodies formation and thus prevention of infliximab efficacy reduction. Case reports, series of patients and trials studying the effect of etanercept and adalimumab on hidradenitis suppurativa were published (15, $16,17,18)$. The results were comparable with infliximab - fast improvement of the disease, but often the recurrency occurs soon, even though cases of long-lasting remission without the need of continuous treatment were described.

Dapsone is considered as one of therapeutical possibilities with good clinical effect in patients with hidradenitis suppurativa. Hidradenitis suppurativa is a disease, which belongs to neutrophil dermatoses. In these the hyperreactive neutrophiles in the area rich with apocrine sweat glands accumulate. Dapson is the drug effectively used mostly in treatment of neutrophile dermatoses. In addition to bacteriostatic effect (which is used in treatment of lepra) it has also the immunomodulation effect - inhibits cytotoxicity and chemotactism of polymorphonuclear leukocytes. Also the neutralizing effect of TNF $\alpha$ is assumed. Neutrophiles, and also TNF $\alpha$ play important role in the pathogenesis of hidradenitis suppurativa. From this point of view dapsone is considered as very appropriate and cheap alternative in treatment of this chronical disease.

Kaur et al $(13,14)$ described the cases of 6 patients with hidradenitis suppurativa treated with dapsone. The dosage varied from 25 to $150 \mathrm{mg} /$ day. All 6 patients profited from therapy, the effect was achieved in 4-12 weeks after the start. All patients required continuous therapy (dapsone dosage varied from 50 to 150 $\mathrm{mg} /$ day) and were followed for the period of 24 months. Therapy was tolerated well in general.

As far as it is known, this is the first known case of combined therapy of TNF $\alpha$ inhibitor infliximab and dapsone in the treatment of hidradenitis suppurativa. Favorable effect of infliximab in continuous therapy of hidradenitis suppurativa was proved in several case reports, maximum of the effect was reached in induction phase of the treatment and decreased with time. One of the possible reasons could be the production of neutralizing antibodies against infliximab and thus decrease of its effect. More authors combined infliximab and methotrexate because of this, which did not lead to significant extension of its clinical effect compared to cases, where infliximab was used in monotherapy. The second possible explanation could be the fact that other, alternative 
pathway in pathogenesis of disease exists and that TNF $\alpha$ is only important effector, but not regulative cytokine. One of the possibilities how to prevent the decrease of clinical effect in treatment with infliximab is shortening the intervals between the doses in the maintenance phase of treatment. The second possibility is to add classic systemic treatment. Methotrexate does not appear as efficient in monotherapy, nor combined with infliximab. Cyclosporine does not belong to common systemic drugs used in therapy of hidradenitis suppurativa and in one case (7) did not bring significant improvement even in combination with infliximab. Systemic corticoids are efficient in this disease, but mostly in higher dosage, which is inappropriate for long term therapy. Lower doses of Prednison in combination with infliximab did not bring success (7). Successive treatment with dapsone in hidradenitis suppurativa monotherapy were described $(13,14)$ and from the other disease treatment (dermatitis herpetiformis, erythema elevatum et diutinum, IgA linear dermatosis, bulous lupus) it is known, that it can be used in long treatment with no significant risk of serious side effects and that they are not contraindication to patients treated with TNF $\alpha$ blocators. Infusion of infliximab induces quick, but only short term improvement in clinical status, which dapson can hold up after achievement of balanced dose by that it is dosed daily and significantly affects neutrophiles and TNF $\alpha$. It seems that this effect could be directly proportional to dose, which has to be adjusted to individual needs and tolerance of patient under strict surveillance.

Dapsone also belongs to cheap and easily reachable therapeutical possibilities. Even though we could reduce the subjective troubles of patient and the CRP levels are in normal levels for 8 weeks, we cannot say that the disease is in remission. It is possible, that if we can hold the CRP levels in normal for long time, we can get the disease under control completely. CRP is not the only one indicator of inflammation in organism. Absolute number of leucocytes and sedimentation are also important inflammatory markers. In our case we managed to improve the number of leucocytes (and also neutrophiles) to normal levels, but sedimentation remains increased. It is possible, that only normalization of all three inflammatory markers will result in complete remission of disease.

\section{Conclusion}

Hidradenitis suppurativa is a disease, which is not easily held under medicament control. TNF $\alpha$ blocators are the new hope in its treatment. Most experience is with infliximab. Even it seems that the short term effect of this biologic is very good and quick, long term retention is not achieved easily. Thus it is required to reach for combinations of biologic treatment together with systemic treatment. Only various appropriate combinations and therapeutical schemes, together with the need of frequent controls and monitoring of laboratory parameters can lead to final success and thus the complete remission can be achieved.

\section{References}

1. Naldi L. Epidemiology. In: Jemec G, Revuz J, Leyden J (Eds). Hidradenitis suppurativa, Vol.1, Springer, 2006, 58-64.

2. Alexis AF, Strober BE. Off-label dermatologic uses of anti-TNF $\alpha$ therapies. J Cutan Med Surg 2005; 9: 296-302.

3. Martinez F, Nos P, Benlloch S et al. Hidradenitis suppurativa and Crohn's disease: response to treatment with infliximab. Inflamm Bowel Dis 2001; 7 (4): 323-326.

4. Katsanos KH, Christodoulou DK, Tsianos EV. Axillary hidradenitis suppurativa successfully treated with infliximab in a Crohn's disease patient. Am J Gastroenterol 2002; 97: 2155-2156.

5. Lebwohl B, Sapadin AN. Infliximab for the treatment of hidradenitis suppurativa. J Am Acad Dermatol 2003; 49: S275-276.

6. Adams DR, Gordon KB, Devenyi AG et al. Severe hidradenitis suppurativa treated with infliximab infusion. Arch Dermatol 2003; 139: $1540-1542$.

7. Sullivan TP, Welsh E, Kerdel FA et al. Infliximab for hidradenitis suppurativa. Br J Dermatol 2003; 149: 1046-1049.

8. Mekkes JR, Bos JD. Long-term efficacy of a single course of infliximab in hidradenitis suppurativa. Br J Dermatol 2007; 158: 370-374.

9. Thielen AM, Barde C, Saurat J-H. Long-term infliximab for severe hidradenitis suppurativa. Br J Dermatol 2006; 154: 1105-1108.

10. Brunasso AMG, Delfino C, Massone C. Hidradenitis suppurativa: are tumor necrosis factor- $\alpha$ blockers the ultimate alternative? Br J Dermatol 2008; 159: 761-763.

11. Grant A, Gonzales T, Montgomery MO et al. Infliximab therapy for patients with moderate to severe hidradenitis suppurativa: A randomized, double-blind, placebo-controlled crossover trial. J Am Acad Dermatol 2009; 62 (2): 205-217.

12. Jemec GB. Methotrexate is of limited value in the treatment of hidradenitis suppurativa. Clin Exp Dermatol 2002; 47: 280-285.

13. Kaur MR, Gupta S, Taibjee S, Lewis HM. Hidradenitis suppurativa treated with dapsone, a case series of six patients. Br J Dermatol 2005; 153 (Suppl 1): 39-40.

14. Kaur MR, Lewis HM. Hidradenitis suppurativa treated with dapsone: A case series of five patients. J Dermatol Treat 2006; 17 (4): 211-213.

15. Moul DK, Korman NJ. Severe hidradenitis suppurativa treated with adalimumab. Arch Dermatol 2006; 142: 1110-1112.

16. Pelekanou A, Kanni T, Savva A et al. Long-term efficacy of etanercept in hidradenitis suppurativa: results from an open-label phase II prospective trial. Exp Dermatol 2010; 19: 538-540.

17. Cusack C, Buckley C. Etanercept: effective in management of hidradenitis suppurativa. Br J Dermatol 2006; 154: 726-729.

18. Zangrilli A, Esposito M, Mio G, Mazzotta A, Chimenti S. Longterm efficacy of etanercept in hidradenitis suppurativa. JEADV 2008; 22: $1260-1262$.

19. van der Zee HH, van der Wounde CI, Florencia EF, Prens EP. Hidradenitis suppurativa and inflammatory bowel disease: are they associated? Results of a pilot study. Br J Dermatol 2010; 162: 195-197.

Received August 24, 2010. Accepted February 14, 2012. 